\title{
PC based control of non linear motor drive
}

K. Alice Mary and P. Ramana

Department of EEE, GMR Institute of Technology, RAJAM - 523127, Srikakulam District, A.P., India

Email: Kalice_mary@yahoo.co.in

\begin{abstract}
Developments in power electronic switches and low-cost computational hardware have made it possible to design and implement sophisticated control strategies for variable speed drives. Such an attempt has been made in this work, for a voltage source inverter fed synchronous motor drive which is absolutely nonlinear in its nature. Certain important issues in design including the speed controller design, generation of reference currents, state feedback linearization and control of the current dynamics, as well as a nonlinear reduced order observer design have been resolved. The proposed scheme is validated through extensive numerical simulations and a laboratory scale implementation.
\end{abstract}

Keywords: Motor drive, non linear, power controller, electronic switches.

\section{Introduction}

Most of the control systems are designed based on a linearized model of the system. Feedback linearization has been applied to only some simplified models, which do not take into account the presence of damper windings. The torque (llic-Spong, 1987) developed by a synchronous motor is a product of different fluxes and currents and this nonlinearity is not linearizable unless one of the components is held constant. The concept of "field orientation" introduced by Blaschke (1972) deals with this nonlinearity by resolving the armature currents into the so called $d$ - and $q$ - axes and forcing the $d$ component of the stator flux to zero (Bayer et al., 1972). However, this results in a lagging power factor operation of the motor. Feedback linearization of the machine model requires the knowledge of the damper winding currents, which are immeasurable. On the other hand, a linear observer (Chattopadhyay \& Meher, 1989) cannot be designed, since the model under consideration is still a nonlinear one. Thus it is necessary to design a nonlinear observer to estimate the states, which can in turn be used for feedback linearization.

In this work, all the above limitations have been overcome (Alice Mary, 1998). The exact linearization of the model of a wound field synchronous motor (WFSM) with damper windings has been carried out. The proposed controller represented in the conventional two-loop structure for the motor drive is shown in Fig. 1. The outer loop is the speed controller, which is a PI controller (Maswood, 1995), the output of which is the reference value of he torque $T_{e}^{*}$. From this value, the reference values of the stator and field currents are computed for a desired internal angle $\psi$ and a desired torque angle $\delta$ (Fig. 2). This gives rise to the flexibility in choosing the power factor of the motor from lagging to leading values including unity. Field oriented control can also be obtained as a special case, by setting the power factor angle to be equal to the torque angle, resulting in complete decoupling between the armature flux and the field flux. The inner loop is the current control loop (Slemon, 1973) which tries to follow the reference currents produced by the outer loop. Further, a nonlinear reduced order observer is designed to estimate the immeasurable damper winding currents.

Motor armature currents in the two phases,

\section{Fig.1.Block copy representation of the proposed} control scheme

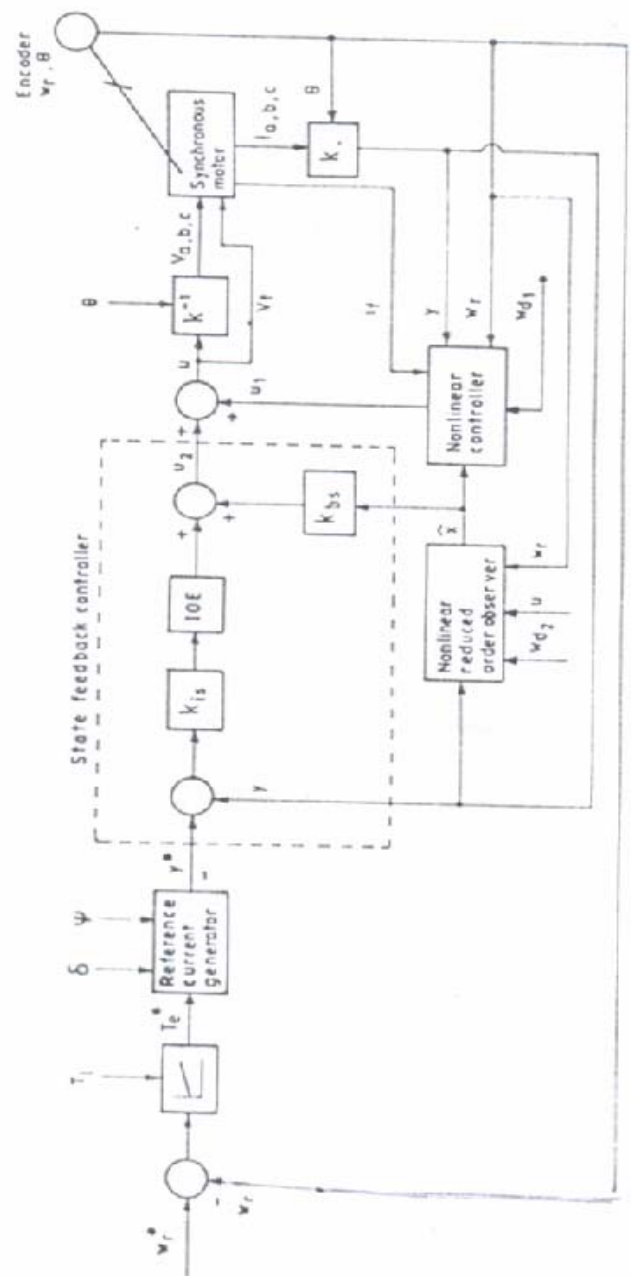


$i_{a}$ and $i_{b}$ and the field current $i_{f r}$ are sensed by Hall effect sensors (HES) using a high performance A/D data acquisition card (PCL-208). The selectable switch is used to set at 16 single ended analog input channels in the bipolar mode. This card uses a 12 bit successive approximation converter (ADC674) to convert analog inputs and the software trigger mode is used for starting $A / D$ conversion. The turns ratio of the hall effect sensor (HES) is 1000:1. A resistor of $660 \Omega$ is connected at the secondary of HES from which the current is sensed and the output is directly connected to the PC through a 20-pin flat ribbon cable. A scaling factor of $1000 / 660=1.515$ is taken into account for the computation of these currents.

\section{Fig.2. Phaser diagram of salient pole synchronous} motor

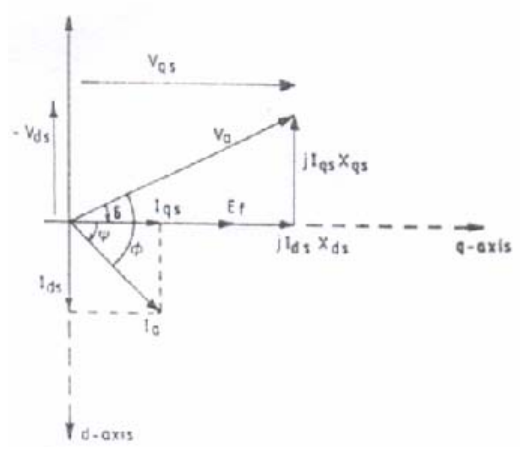

Fig.3. Experimental results for change in speed from $8 \pi$ to $12 \pi \mathrm{rad} / \mathrm{s}$ for field oriented control a) Simulation b) Experimental
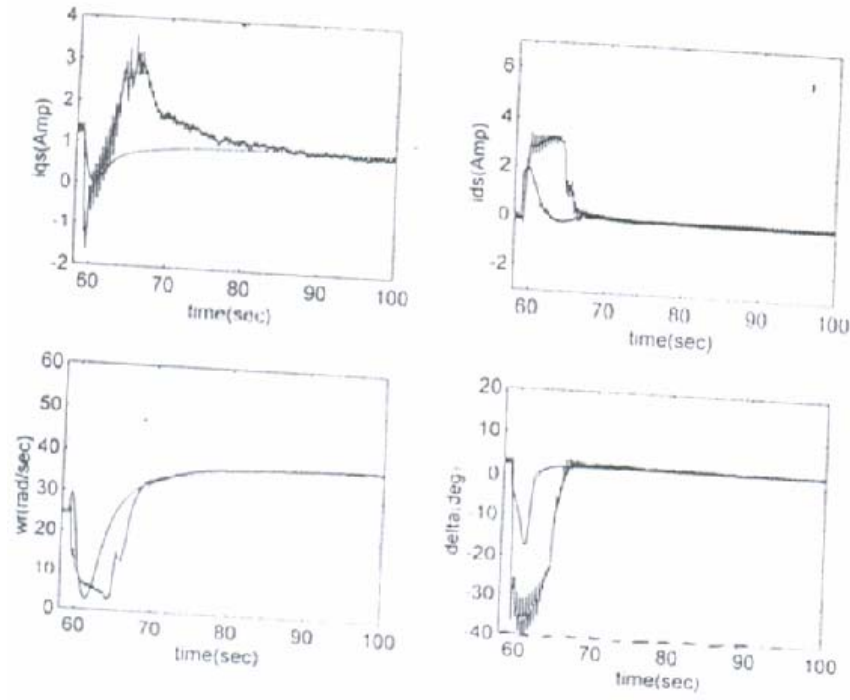

A high resolution incremental encoder along with a timer/event card (PCL-223) is used to obtain rotor position angle information. An INTEL 8253 chip is used for the speed and rotor position measurement. Counters \#0 and \#1 are initialized in mode \#0 and counter \#2 in mode \#1. Counter \#0 generates the standard clock frequency for the encoder speed measurement. $K_{1}$ of encoder, which gives 2500 pulses/rev, is connected to the clocks of counters \#1 and \#2 and $K_{0}$, the marker pulse signal is connected to the gate of counter \#2. A clock frequency of $250 \mathrm{kHz}$ is selected by a suitable address register. The output of counter \#1 gives the speed measurement and the output of counter \#2 give the rotor position angle. The above circuits are fabricated in printed circuit boards.

The design methodology

The voltage equations for the synchronous motor in rotor reference frame are

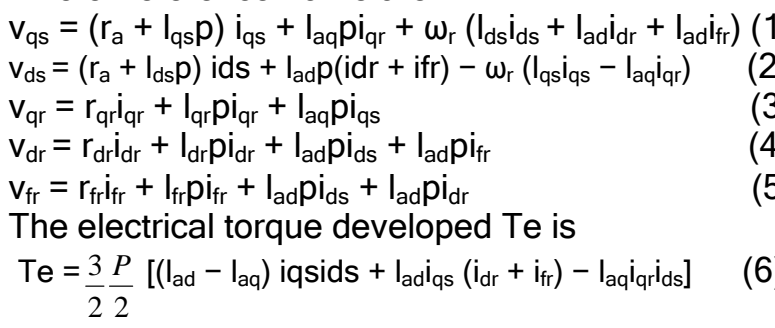

The torque balance equation is

$\frac{2}{P} J p \omega_{r}=T_{e}-T_{l}-\frac{2}{P} \beta \omega_{r}$

Where all voltages $(v)$ and currents(i) refer to the rotor reference frame. The subscripts qs, ds, qr and $\mathrm{dr}$ corresponds to $\mathrm{q}$ and $\mathrm{d}$ axis quantities for the stator(s) and rotor( $r$ ) in all combinations; $r_{a}$ denotes the armature resistance and $\mathrm{I}_{\mathrm{fr}}$ the field inductance; while other inductances are denoted by $I_{q s}, I_{d s}$ etc. $T_{e}$ is the developed torque. The rotor speed is given by $\omega_{r}$ and the load torque by $T_{1}$. $J$ is moment of inertia, $P$ is the number of poles and $\beta$ is the coefficient of viscous friction. The derivative operator is represented by the symbol, $\mathrm{p}$.

The control scheme

Design of the PI controller

The output of the PI controller is the reference torque from which the reference currents $i_{\mathrm{qs}}{ }^{*}, \mathrm{i}_{\mathrm{ds}}{ }^{*}$ and $\mathrm{i}_{\mathrm{fr}}{ }^{*}$ for stator and field winding can be generated. The controller equation for reference torque is

$$
T_{e}^{*}=k_{p} e+k_{i} \int_{0}^{t} e d t
$$

where

$$
e=\omega_{e}-\omega_{r}
$$

$\omega_{e}$ is the set (reference) speed and $\omega_{r}$ is the actual speed of the rotor and $k_{p}$ and $k_{i}$ are the proportional and integral gains of the controller respectively. They are obtained as,

$k_{i}=\frac{J}{2} \omega_{n}^{2}$ 
Indian Journal of Science and Technology

$$
k_{p}=J \varsigma \omega_{n}-\frac{\beta}{2}
$$

where $\zeta=$ desired value of damping ratio, and $\omega_{n}=$ desired value of natural frequency. The value of $\zeta$ is usually determined from the requirement of permissible maximum overshoot and the un damped natural frequency $\omega_{n}$ determines the time response.

\section{Determination of reference currents for desired $\psi$} and $\delta$

For a given reference torque, $\mathrm{T}_{\mathrm{e}}{ }^{*}$, we obtain

$$
i_{q s}^{*}=\sqrt{\frac{T_{e}^{*} \omega_{r} \tan \delta}{3 \omega_{r} l_{q s}(\tan \psi \tan \delta-1)+3 r_{a}(\tan \delta+\tan \psi)}}
$$

Fig.4. Experimental results for field oriented control showing a) rotor position angle $(\theta) b$ ) control voltage generated by the controller

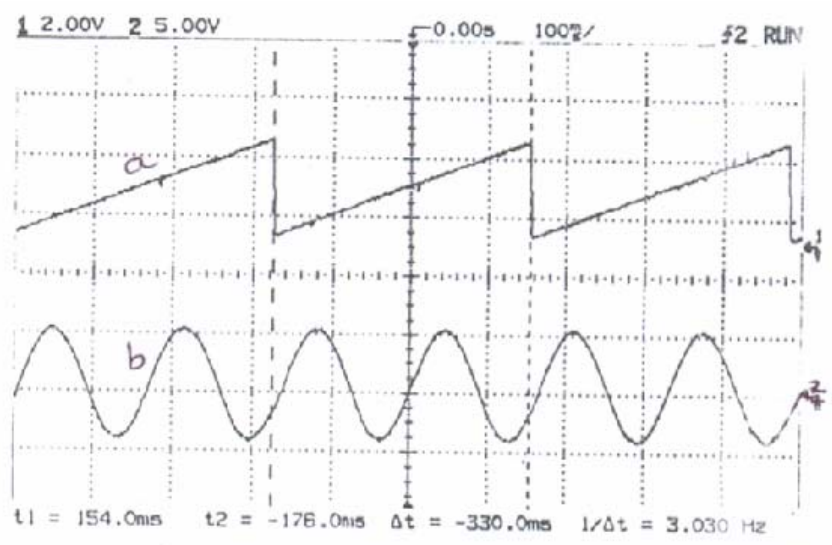

The values of $\mathrm{i}_{\mathrm{ds}}{ }^{*}$ and $\mathrm{i}_{\mathrm{fr}}$ "are obtained by substituting this value of $\mathrm{i}_{\mathrm{qs}}$ in its corresponding equations. However, $\psi$ and $\delta$ should be so chosen that these currents remain real-valued.

Field oriented control

Field oriented (FO) control can be achieved for the wound field (WF) synchronous motor by setting the internal angle $\psi$ to zero in $\mathrm{i}_{\mathrm{ds}}$ equation. This implies that the terminal power factor angle, $\Phi$ is equal to the torque angle, $\delta$

\section{Feedback linearization}

The system model using only the voltage equations (i.e., the electrical subsystem) is expressed as

$x=A x+B u$

Partitioning $A$ into $A$ ' \& $A$

$$
\dot{x}=\left(A^{\prime}+\omega_{r} A^{\prime \prime}\right) x+B u
$$

where $A^{\prime} \& A^{\prime \prime}$ are appropriate matrices [1]. We choose a feedback control law of the form, $\mathrm{u}=\mathrm{u}_{2}+\mathrm{u}_{1}$

$$
\text { http://www.indjst.org }
$$

Vol.1 No.2 (Dec. 2007) where $u_{1}$ and $u_{2}$ are the input control vectors of the nonlinear and linear parts respectively. The nonlinear feedback control law is chosen as

$\mathrm{u}_{1}=\omega_{\mathrm{r}} \mathrm{K}_{1} \mathrm{x}$

where $K_{1}$ is the feedback gain matrix.

In order to get exact cancellation of the nonlinear term,

$$
\begin{aligned}
& A "+\mathrm{BK}_{1}=0 \\
& \text { or, }
\end{aligned}
$$

$A^{\prime \prime}=-\mathrm{BK}_{1}$

If $\mathrm{K}_{1}$ is taken as

$K_{1}=\left[\begin{array}{ccccc}0 & l_{d s} & 0 & l_{a d} & l_{a d} \\ -l_{q s} & 0 & -l_{a q} & 0 & 0 \\ 0 & 0 & 0 & 0 & 0\end{array}\right]$

$x=A^{\prime} x+B u_{2}$

Alternatively, one can choose,

$\mathrm{u}=\left(\omega_{\mathrm{r}}-\omega_{\mathrm{d} 1}\right) \mathrm{K}_{1} \mathrm{x}+\mathrm{u}_{2}$

where $\omega_{\mathrm{d} 1}$ is a design constant, which can be chosen for a tradeoff between the linear and nonlinear components of the control signal. To obtain asymptotic following of the reference currents $y_{r}$ via state and integral output feedback,

$u_{2}=K_{b s} x+K_{i s} \int_{0}^{t}\left(y-y_{r}\right) d t$

A reduced order observer is designed to estimate the damper winding currents

$$
\dot{\hat{\zeta}}=D \hat{\zeta}+G u+F y^{\prime}
$$

where,

$D=$ observer system matrix,

$\mathrm{G}=$ input (control) matrix, and

$\mathrm{F}=$ input (output) matrix.

\section{Software-realization}

\section{Transformation of the axes}

The line currents sensed are converted to d-q axes components using Park's transformation for the purpose of control signal computations. The generated control voltages in $d-q$ axes are converted into a-b-c axes using the inverse transformation and outputted as voltage commands to the voltage source inverter. The transformation can be represented as follows:

$$
i_{q-d}=K i_{a-b-c}
$$

where,

$$
\begin{aligned}
& i_{a-b-c}=\left[\begin{array}{lll}
i_{a} & i_{b} & i_{c}
\end{array}\right]^{T} \\
& i_{q-d}=\left[\begin{array}{lll}
i_{q s} & i_{d s} & i_{o s}
\end{array}\right]^{T}
\end{aligned}
$$


Indian Journal of Science and Technology

$$
K=\frac{2}{3}\left[\begin{array}{ccc}
\cos \theta & \cos \left(\theta-120^{\circ}\right) & \cos \left(\theta-240^{\circ}\right) \\
\sin \theta & \sin \left(\theta-120^{\circ}\right) & \sin \left(\theta-240^{\circ}\right) \\
1 / 2 & 1 / 2 & 1 / 2
\end{array}\right]
$$

and

$v_{a-b-c}=K^{-1} v_{q-d}$

where,

$$
\begin{aligned}
& v_{a-b-c}=\left[\begin{array}{lll}
v_{a} & v_{b} & v_{c}
\end{array}\right]^{T} \\
& v_{q-d}=\left[\begin{array}{lll}
v_{q s} & v_{d s} & v_{0 s}
\end{array}\right]^{T}
\end{aligned}
$$

$$
K^{-1}=\left[\begin{array}{ccc}
\cos \theta & \sin \theta & 1 \\
\cos \left(\theta-120^{\circ}\right) & \sin \left(\theta-120^{\circ}\right) & 1 \\
\cos \left(\theta-240^{\circ}\right) & \sin \left(\theta-240^{\circ}\right) & 1
\end{array}\right]
$$

\section{Discretization of the feedback control law}

For the purpose of digital implementation, the continuous-time controller and observer equations developed in section 5 are converted into discrete form as follows. The integral part of the controller equation in discrete form is approximated as,

$\int_{0}^{T}\left(y-y_{r}\right) d t \cong T \sum\left(y(k T)-y_{r}(k T)\right)$

where $\mathrm{T}$, the sampling time $=1 \mathrm{~ms}$ and $\mathrm{k}=$ $0,1,2,3 . \ldots \mathrm{n}$ Thus, the control signal is computed by,

$$
\begin{aligned}
u(n T)= & K_{b s} x(n T)+K_{i s} T \sum_{k=1}^{n-1}\left(y(k T)-y_{r}(k T)\right) \\
& +\left(\omega_{r}-\omega_{d 1}\right) K_{1} x(n T)
\end{aligned}
$$

\section{Discretization of observer equation}

For sufficiently small $\mathrm{T}$, the discretised form of the observer system equation is

$$
\hat{\zeta}(n+1) T=\hat{\zeta}(n T)+T D \hat{\zeta}(n T)+T G u(n T)+T F y^{\prime}(n T)
$$

and

$$
y^{\prime}(n T)=C^{\prime} x(n T)
$$

\section{Real-time implementation}

The equations are in a form suitable for realization in a digital computer. The software is implemented using a high level language, Turbo- $C$ with the necessary subroutines supporting l/O cards.

The steps involved are:

(1) Initialize the program variables, I/O cards and counters.

(2) Read the gain matrices $\mathrm{K}_{\mathrm{bs}}, \mathrm{K}_{\mathrm{is}}, \mathrm{K}_{1}, \mathrm{D}, \mathrm{G}, \mathrm{F}$.

(3) For time $=0.0 \mathrm{sec}$, and final time $=300 \mathrm{sec}$, do

(3.1) Get current measurements from PCL-208.

(3.2) Transform currents from a-b-c to d-q axes.

(3.3) Get counts from8253 counters.

(3.4) Compute speed and rotor angle $(\theta))$ from the count values.

(3.5) Calculate reference torque and currents http://www.indjst.org

Vol.1 No.2 (Dec. 2007)

(3.6) Compute the estimates of idr and $\mathrm{i}_{\mathrm{qr}}$.

(3.7) Compute control voltages.

(3.8) Convert voltages from d-q to a-b-c axes.

(3.9) Output reference voltages and display signals through PCL-726.

(3.10) Save variables in memory.

(4) Reset the output channels.

The time required by the PC to execute the loop once has been found to be about $385 \mu \mathrm{sec}$.

Results and discussion

The experimental drive system is subjected to different tests. First of all, the synchronous motor is started in open loop

Fig.5. Experimental results for field oriented control showing line currents of the motor a)ib b)ia

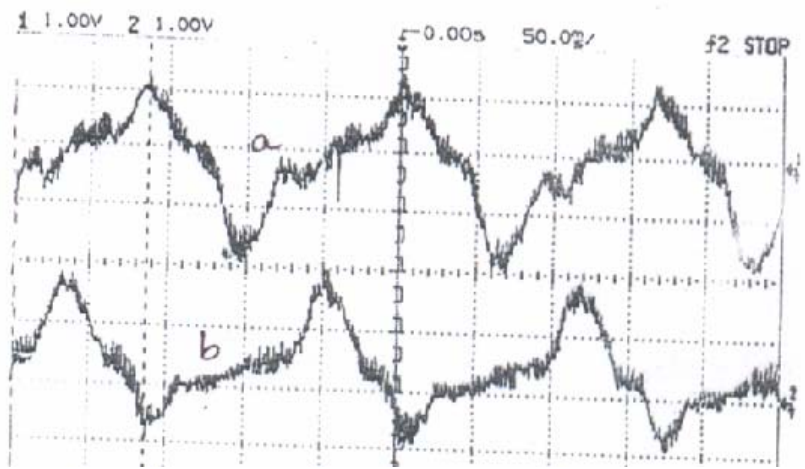

condition by giving a frequency of $2 \mathrm{~Hz}$ and generating sinusoidal voltages through the analog output channels. The supply frequency is changed to $4 \mathrm{~Hz}$ after $30 \mathrm{sec}$, and the open loop step responses are obtained. Another set of data is generated for a sudden change in load torque from $2 \mathrm{~N}-\mathrm{m}$ to $3 \mathrm{~N}-\mathrm{m}$. The change in load torque is achieved by coupling a dc generator to the motor and suddenly switching on a resistive load.

For closed loop operation, the machine is initially started in open loop and taken to a speed of $8 \pi \mathrm{rad} / \mathrm{s}$ (in electrical angle, corresponding to a frequency of $4 \mathrm{~Hz}$ ). After about $60 \mathrm{sec}$, the speed reference is changed to $12 \pi \mathrm{rad} / \mathrm{s}$ and the control algorithm is switched to closed loop operation. The observer is, however, started at time $\mathrm{t}=0$, so that at the time of closure of the loop, the converged values of the estimates of the damper currents are available. It should be mentioned here, that although the synchronous motor used is a wound field one, all the experimental runs are performed with a constant field excitation.

Experimental traces of the rotor position angle $(\theta)$ and the control voltage waveform, $v_{a}$ are shown in Fig 3 under the field oriented control. The first trace clearly indicates that the mechanical speed of the motor corresponds to about $3 \mathrm{~Hz}$, corresponding to the electrical speed of $12 \pi \mathrm{rad} / \mathrm{s}$, which is equal to the reference value. Finally, 
Indian Journal of Science and Technology

figures 4 and 5 show the waveforms of the two line currents, $i_{a}$ and $i_{b}$ relative to each other and along with the position angle $(\theta)$.

\section{Conclusions}

The designed controller and observer for the VSISM drive system has been implemented with the help of a personal computer (PC) with analog $\mathrm{I} / \mathrm{O}$ and timer/counter modules along with interfacing circuits as needed and tested in the laboratory environment. The various components of the control circuit including interfacing circuits needed for measurement and data acquisition have been fabricated and individually tested before integrating into a complete setup. The controller software needed to implement the nonlinear control laws and the nonlinear reduced order observer are developed in a high level language. The dynamic responses of the drive system are obtained after tests in the laboratory and they agree well with the simulation results within the limitations of the experimental set-up and other assumptions in the simulation.

\section{References}

1. Alice Mary K (1998) Nonlinear Modelling, Control System Design and Implementation for an Inverter-fed Synchronous Motor Drive. Ph.D. Thesis, Department of Electrical Engineering, I.I.T. Kharagpur. http://www.indjst.org Vol.1 No.2 (Dec. 2007)

2. Bayer $\mathrm{KH}$, Waldmann $\mathrm{H}$ and Weibelzahl $\mathrm{M}$ (1972) Field oriented closed-loop control of a synchronous machine with the new TRANSVECTOR control system. Siemens Rev. 39, 217-220.

3. Blaschke F (1972') The principle of field orientation as applied to the new TRANSVECTOR closed-loop control system for rotating field machines. Siemens Rev. 39, 217-220.

4. Chattopadhyay AK and Meher N (1989) Microprocessor implementation of a state feedback control strategy for a current source inverter fed induction motor drive. IEEE Trans. Power. Elect. 4, 279-288.

5. llic-Spong M, Miller TJE, Macminn SR and Thorp J S (1987) Instantaneous torque control of electronic motor drives. IEEE Trans. Power Elect. PE-2, 55-61.

6. Maswood Al (1995) A PWM voltage source inverter with $\mathrm{PI}$ controller for instantaneous motor current control. IEEE Catalogue no-95, 8025, pp:834-837.

7. Slemon GR, Forsythe JB and Dewan SB (1973) Controlled power angle synchronous motor inverter drive system. IEEE Trans. Ind. Appl. IA-9, 216-219. 